University of Wollongong

Research Online

Australian Institute for Innovative Materials -

Papers

Australian Institute for Innovative Materials

2009

Generation of terahertz radiation by bulk and surface optical rectification from crystal planes of arbitrary orientation

Stuart Hargreaves

University of Wollongong

Krunal Radhanpura

University of Wollongong, krunal@uow.edu.au

Roger A. Lewis

University of Wollongong, roger@uow.edu.au

Follow this and additional works at: https://ro.uow.edu.au/aiimpapers

Part of the Engineering Commons, and the Physical Sciences and Mathematics Commons

Research Online is the open access institutional repository for the University of Wollongong. For further information contact the UOW Library: research-pubs@uow.edu.au 


\title{
Generation of terahertz radiation by bulk and surface optical rectification from crystal planes of arbitrary orientation
}

\author{
Abstract \\ The theory of second-order nonlinear bulk and third-order nonlinear surface electric-field-induced optical \\ rectification is developed for zinc-blende $4^{-} 3 \mathrm{~m}$ crystal faces of arbitrary indices (hkl). The theory is then \\ used to account for our experimental results for terahertz generation from GaAs high-index planes (11N)A \\ and $(11 \mathrm{~N}) \mathrm{B}$, where $\mathrm{N}=2,3,4$, and 5 . The data combined with the calculations allow us to demonstrate that \\ a surface field is present on both the Ga-rich and As-rich faces and to determine that the signs are \\ opposite and that the strengths are similar. Such conclusions are inaccessible by work restricted to low- \\ index planes. \\ Disciplines \\ Engineering | Physical Sciences and Mathematics

\section{Publication Details} \\ Hargreaves, S., Radhanpura, K. \& Lewis, R. A. (2009). Generation of terahertz radiation by bulk and surface \\ optical rectification from crystal planes of arbitrary orientation. Physical Review B (Condensed Matter and \\ Materials Physics), 80 (19), 195323-1-195323-16.
}




\title{
Generation of terahertz radiation by bulk and surface optical rectification from crystal planes of arbitrary orientation
}

\author{
S. Hargreaves, K. Radhanpura, and R. A. Lewis \\ Institute for Superconducting and Electronic Materials, University of Wollongong, Wollongong, New South Wales 2522, Australia
}

(Received 20 June 2009; revised manuscript received 22 October 2009; published 30 November 2009)

\begin{abstract}
The theory of second-order nonlinear bulk and third-order nonlinear surface electric-field-induced optical rectification is developed for zinc-blende $\overline{4} 3 m$ crystal faces of arbitrary indices $(h k l)$. The theory is then used to account for our experimental results for terahertz generation from GaAs high-index planes $(11 N) A$ and $(11 N) B$, where $N=2,3,4$, and 5 . The data combined with the calculations allow us to demonstrate that a surface field is present on both the Ga-rich and As-rich faces and to determine that the signs are opposite and that the strengths are similar. Such conclusions are inaccessible by work restricted to low-index planes.
\end{abstract}

DOI: 10.1103/PhysRevB.80.195323

PACS number(s): 78.20.Bh, 78.30.Fs, 78.40.Fy

\section{INTRODUCTION}

The generation of coherent terahertz $(\mathrm{THz})$ frequency electromagnetic radiation by optical excitation using short pulses of near-infrared (NIR) radiation has progressed rapidly in the past two decades. ${ }^{1,2}$ Several mechanisms are known that mediate the conversion of NIR to $\mathrm{THz}$ radiation. The first to be widely exploited was the photoconductivity (PC) mechanism, technologically realized by applying an external potential to a semiconductor surface via an appropriate electrode structure, such as an "Auston switch,"3 strip-line antenna, ${ }^{4}$ logarithmic-periodic antenna, ${ }^{5}$ or Ag-paint dots. ${ }^{6,7}$ The PC mechanism will not be discussed further. Turning now to mechanisms that do not involve electrodes on the sample, these may be separated into two main classes: transient current (TC) mechanisms and optical rectification (OR) ${ }^{8,9}$ The TC effects may in turn be divided into those arising from the acceleration of charge carriers by band bending at the semiconductor surface, called surface-field (SF) effects, ${ }^{10}$ and those arising from different rates of diffusion of the (photogenerated) electrons and holes in the material, the photo-Dember (PD) effect. ${ }^{11}$ In many cases the TC and $\mathrm{OR}$ processes occur simultaneously. TC effects tend to saturate at high optical fluences whereas OR effects do not. ${ }^{12}$ This in some cases allows the contribution of the two mechanisms to be distinguished. A practical consequence is that OR emitters benefit more from higher pump powers than TC emitters. ${ }^{12}$ The definitive test for distinguishing TC and OR mechanisms, however, rests on geometrical considerations. Rotating the crystal about its surface normal often leads to a systematic variation in the $\mathrm{THz}$ emission by OR but to no change in the THz emission by TC. We now concentrate on $\mathrm{OR}$ and the role that the experimental geometry plays in determining the amount of $\mathrm{THz}$ radiation emitted.

Early experiments on $\mathrm{THz}$ generation by OR employed simple geometries. The simplest is transmission geometry. ${ }^{13-16}$ The excitation laser beam strikes the crystal at normal incidence and the generated $\mathrm{THz}$ field is detected in the straight-through direction. It turns out that there is no bulk OR in the case of (100) crystals in this geometry, but (110) and (111) crystals give distinctive patterns of radiation as the azimuthal angle is varied. ${ }^{15}$ A more complicated arrangement is reflection geometry. ${ }^{11,17} \mathrm{An}$ in-plane component of the optical excitation field now leads to the generation of $\mathrm{THz}$ radiation by (100) crystals. A practical complication for many materials is that the refractive index at the pump frequency, $n_{\mathrm{NIR}}$, differs from the refractive index at the emission frequency, $n_{\mathrm{THz}}$. Hence "specular" reflection, which means the angles of incidence and reflection are equal, will correspond to different refraction angles for the NIR and $\mathrm{THz}$ radiation. The situation is described as "quasireflection."

The theory of second-order bulk OR has been developed and compared with experiments for simple crystal faces, such as (100), (110), (111), ${ }^{15,17,18}$ and, recently, (112). ${ }^{19}$ Fewer works have considered the third-order surface-fieldinduced OR, but theory and data again have been presented for (100), (110), (111), and (112) faces. ${ }^{18,19}$ With some exceptions, the previous work has been concerned with the overall pattern of the variation in $\mathrm{THz}$ field as the azimuthal angle varies and has not paid close attention to, for example, the relation of the polarization components at a particular azimuthal angle, or the exact in-plane direction from which the azimuthal angle is measured or the sense of the rotation. Moreover, the coordinate systems used to represent the crystal axes, the laboratory frame, and so on, have been proliferated by different authors for different geometries. Here we present a cohesive general theory. The incidence beam may be oriented arbitrarily with respect to the crystal surface; the crystal surface may have arbitrary Miller indices; the detection is at an arbitrary position. Our interest is in the conclusions that may be drawn from symmetry considerations alone and not in the specific microscopic mechanisms that depend on such factors as the materials parameters and energy and intensity of the excitation radiation and have been the subject of previous works. ${ }^{20,21}$ Related work deals with the reduction in symmetry provided by quantum wells. ${ }^{22}$

After presenting the general theory, we illustrate its use in interpreting our experimental data for the orthogonally polarized components of the $\mathrm{THz}$ emission from (112)A, (113)A, (114) $A,(115) A,(112) B,(113) B,(114) B$, and (115) $B$ faces of GaAs in transmission geometry. The suffixes $A$ and $B$ denote Ga-rich and As-rich faces, respectively. High-index planes have been shown to have attractive electronic properties. ${ }^{23,24}$ The determination of the surface reconstructions has been made by scanning tunneling microscopy for GaAs (111)A 


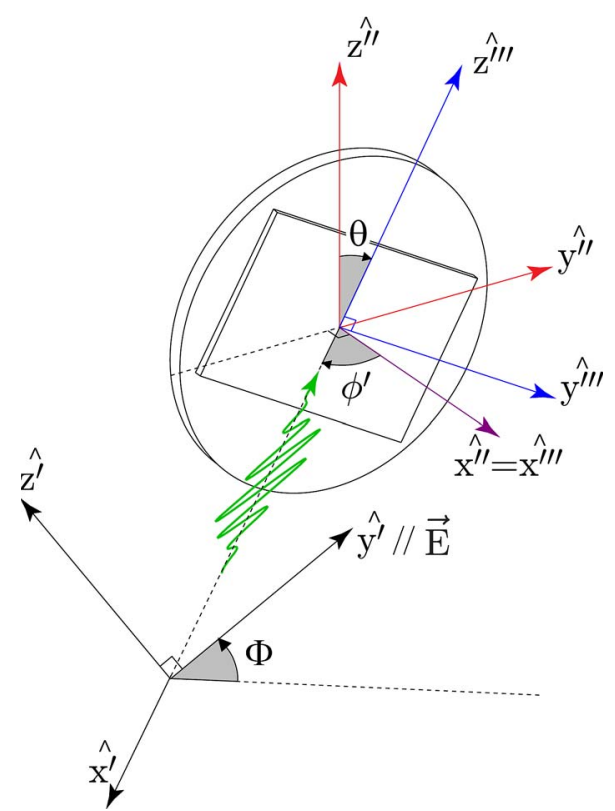

FIG. 1. (Color online) Coordinate axes and angles used. The incident radiation propagates in the $-\widehat{\mathbf{x}^{\prime}}$ direction and is polarized in the $\widehat{\mathbf{y}^{\prime}}$ direction. The outward-pointing normal to the crystal surface is given by $\widehat{\mathbf{x}^{\prime \prime}}$. The triple-primed axis system rotates with the crystal. The angle of incidence is $\phi^{\prime}$. The angle of polarization is $\Phi$. The azimuthal angle is $\theta$.

and $B$ faces, ${ }^{25}$ the (311) $A$ face ${ }^{26,27}$ and $B$ face,,$^{28}$ among others. We also compare $\mathrm{THz}$ emission from this series with $\mathrm{THz}$ emission from (110) $A$ and (110) $B$ crystals. We are then able to draw conclusions regarding the surface fields at the $A$ and $B$ faces of GaAs. These direct and specific conclusions are only realizable by using high-index crystals; that is, they are not attainable using (100), (110), or (111) crystals.

\section{THEORY}

Figure 1 is provided to assist in picturing some of the coordinate axes and angles employed in developing the theory.

\section{A. Excitation radiation: $\widehat{x^{\prime}}, \widehat{y^{\prime}}$, and $\widehat{z^{\prime}}$}

We assume the excitation radiation is linearly polarized. The radiation may be propagating in any direction in space. We use the properties of the excitation radiation to define a coordinate system specified by unit vectors $\widehat{\mathbf{x}^{\prime}}, \widehat{\mathbf{y}^{\prime}}$, and $\widehat{\mathbf{z}^{\prime}}$. We first define $\widehat{\mathbf{y}^{\prime}}$ to lie in the direction of the polarization of the excitation radiation. Introducing $E_{0}$ to represent the magnitude of the electric field, the electric-field vector is then $\mathbf{E}=E_{0} \widehat{\mathbf{y}^{\prime}}$. In alternative notations we may write $\mathbf{E}$ $=\left(E_{x^{\prime}}, E_{y^{\prime}}, E_{z^{\prime}}\right)=\left(0, E_{0}, 0\right)=(0,1,0) E_{0}$ or

$$
\mathbf{E}=\left[\begin{array}{c}
E_{x^{\prime}} \\
E_{y^{\prime}} \\
E_{z^{\prime}}
\end{array}\right]=\left[\begin{array}{c}
0 \\
E_{0} \\
0
\end{array}\right]=\left[\begin{array}{l}
0 \\
1 \\
0
\end{array}\right] E_{0} .
$$

We next define $\widehat{\mathbf{x}^{\prime}}$ to lie in the direction opposite to the direction of propagation. (This choice proves convenient when we later consider the excitation radiation in relation to the surface normal of the crystal.) Finally, we use conventional right-hand triads in this work, and so define $\widehat{\mathbf{z}^{\prime}}$ by $\widehat{\mathbf{z}^{\prime}}$ $=\widehat{\mathbf{x}^{\prime}} \times \widehat{\mathbf{y}^{\prime}}$.

\section{B. Incidence angle: $\phi^{\prime}$}

The excitation radiation strikes the crystal sample at an arbitrary angle of incidence $\phi^{\prime}$. The outward-directed normal to the crystal surface will define the unit vector $\widehat{\mathbf{x}^{\prime \prime}}$. For nonnormal incidence, $\phi^{\prime} \neq 0$, the plane in which $\widehat{\mathbf{x}^{\prime}}$ and $\widehat{\mathbf{x}^{\prime \prime}}$ lie defines the plane of reflection. We then define the unit vector $\widehat{\mathbf{z}^{\prime \prime}}$ to lie perpendicular to this plane in the direction of $\widehat{\mathbf{x}^{\prime}}$ $\times \widehat{\mathbf{x}^{\prime \prime}}$. For normal incidence, $\phi^{\prime}=0$, we define $\widehat{\mathbf{z}^{\prime \prime}}=\widehat{\mathbf{z}^{\prime}}$. Finally in this triad we define the unit vector $\widehat{\mathbf{y}^{\prime \prime}}$ by $\widehat{\mathbf{y}^{\prime \prime}}=\widehat{\mathbf{z}^{\prime \prime}} \times \widehat{\mathbf{x}^{\prime \prime}}$. The three unit vectors may be imagined to be fixed to the surface, with $\widehat{\mathbf{y}^{\prime \prime}}$ and $\widehat{\mathbf{z}^{\prime \prime}}$ lying in the surface, respectively in the reflection plane and perpendicular to it, and $\widehat{\mathbf{x}^{\prime \prime}}$ perpendicular to the surface.

At the surface, refraction occurs according to the generalized Snell's law:

$$
n_{\text {air,NIR }} \sin \phi^{\prime}=n_{\text {crystal,NIR }} \sin \phi_{\text {NIR }} .
$$

Here $\phi_{\text {NIR }}$ is the angle of refraction in the crystal, $n_{\text {air,NIR }}$ is the refractive index of the ambient atmosphere at the pump photon frequency, and $n_{\text {crystal,NIR }}$ is the index of the crystal at this photon frequency.

\section{Polarization angle: $\Phi$}

In general, the polarization of the excitation radiation may be at any orientation with respect to the crystal. We define the polarization angle $\Phi$ to be the angle that the polarization direction of the incident radiation $\widehat{\left(\mathbf{y}^{\prime}\right)}$ makes with the $\widehat{\mathbf{x}^{\prime \prime}}-\widehat{\mathbf{y}^{\prime \prime}}$ plane. This plane is perpendicular to the crystal surface and, in the case of non-normal incidence, is the reflection plane. We specify the sense of $\Phi$ to be positive for a clockwise rotation with respect to the direction $\widehat{\mathbf{x}^{\prime}}$, with $\Phi=0$ corresponding to $\widehat{\mathbf{z}^{\prime}}$ being parallel to $\widehat{\mathbf{z}^{\prime \prime}}$. (For normal incidence we have already $\widehat{\mathbf{x}^{\prime \prime}}=\widehat{\mathbf{x}^{\prime}}$ and $\widehat{\mathbf{z}^{\prime \prime}}=\widehat{\mathbf{z}^{\prime}}$, so $\widehat{\mathbf{y}^{\prime \prime}}=\widehat{\mathbf{y}^{\prime}}$ and $\Phi=0$.)

We may identify $p$ and $s$ components of the excitation radiation according to the usual meaning,

$$
\left[\begin{array}{c}
E_{p} \\
E_{s}
\end{array}\right]=\left[\begin{array}{c}
\cos \Phi \\
\sin \Phi
\end{array}\right] E_{0} .
$$

For normal incidence, the "reflection plane" is not defined, but we will still refer to the $p$ and $s$ components of the incident beam as given by Eq. (3). So for normal incidence $E_{p}=E_{0}$ and $E_{s}=0$.

\section{Coordinate system fixed to the crystal surface: $\widehat{\mathbf{x}^{\prime \prime}}, \widehat{\mathbf{y}^{\prime \prime}}$, and $\widehat{\mathbf{z}^{\prime \prime}}$}

Taking into account the angle of incidence $\phi^{\prime}$ and the angle of polarization $\Phi$, the electric field of the excitation 
radiation $E_{0} \widehat{\mathbf{y}^{\prime}}$ in the double-primed coordinate system attached to the crystal surface is

$$
\left[\begin{array}{c}
E_{x^{\prime \prime}} \\
E_{y^{\prime \prime}} \\
E_{z^{\prime \prime}}
\end{array}\right]=\left[\begin{array}{ccc}
+\cos \phi_{\mathrm{NIR}} & +\sin \phi_{\mathrm{NIR}} & 0 \\
-\sin \phi_{\mathrm{NIR}} & +\cos \phi_{\mathrm{NIR}} & 0 \\
0 & 0 & 1
\end{array}\right]\left[\begin{array}{c}
0 \\
\cos \Phi \\
\sin \Phi
\end{array}\right] E_{0} .
$$

On multiplying the matrices we may express the result as

$$
\left[\begin{array}{c}
E_{x^{\prime \prime}} \\
E_{y^{\prime \prime}} \\
E_{z^{\prime \prime}}
\end{array}\right]=\left[\begin{array}{c}
\sin \phi_{\mathrm{NIR}} \cos \Phi \\
\cos \phi_{\mathrm{NIR}} \cos \Phi \\
\sin \Phi
\end{array}\right] \quad E_{0}=\left[\begin{array}{c}
\sin \phi_{\mathrm{NIR}} \\
\cos \phi_{\mathrm{NIR}} \\
\tan \Phi
\end{array}\right] \cos \Phi E_{0} .
$$

\section{E. Azimuthal angle: $\theta$}

To account for rotation of the crystal about its surface normal, the azimuthal angle $\theta$ is introduced. To facilitate the working, we define a second coordinate system fixed to the crystal. We denote this the triple-primed system, being the double-primed system rotated counterclockwise about the $\widehat{\mathbf{x}^{\prime \prime}}=\widehat{\mathbf{x}^{\prime \prime \prime}}$ axis through an angle $\theta$. Transforming from the double-primed to triple-primed coordinates yields

$$
\begin{aligned}
{\left[\begin{array}{l}
E_{x^{\prime \prime \prime}} \\
E_{y^{\prime \prime \prime}} \\
E_{z^{\prime \prime \prime}}
\end{array}\right]=} & {\left[\begin{array}{ccc}
1 & 0 & 0 \\
0 & +\cos \theta & -\sin \theta \\
0 & +\sin \theta+\cos \theta
\end{array}\right]\left[\begin{array}{c}
E_{x^{\prime \prime}} \\
E_{y^{\prime \prime}} \\
E_{z^{\prime \prime}}
\end{array}\right] } \\
= & \left.\begin{array}{c}
\sin \phi_{\mathrm{NIR}} \cos \Phi \\
\cos \theta \cos \phi_{\mathrm{NIR}} \cos \Phi-\sin \theta \sin \Phi \\
\sin \theta \cos \phi_{\mathrm{NIR}} \cos \Phi+\cos \theta \sin \Phi
\end{array}\right] E_{0} .
\end{aligned}
$$

\section{F. Crystal axes: $\hat{\mathbf{x}}, \hat{\mathbf{y}}$, and $\hat{\mathbf{z}}$}

The principal axes of the crystal define the unit vectors $\hat{\mathbf{x}}$, $\hat{\mathbf{y}}$, and $\hat{\mathbf{z}}$. The surface of the crystal that the excitation radiation strikes is denoted by Miller indices $h, k$, and $l$. In other words, the vector $h \hat{\mathbf{x}}+k \hat{\mathbf{y}}+l \hat{\mathbf{z}}$ is normal to the surface. The rotation of the crystal about the surface normal is accounted for by the azimuthal angle, but we have so far not specified a crystal direction from which this rotation is measured. We

make the choice that the direction previously specified by $\mathbf{z}^{\prime \prime \prime}$ will lie along the projection of the crystal $\hat{\mathbf{z}}$ axis onto the crystal surface. [This choice is not possible if the crystal $\hat{\mathbf{z}}$ axis is normal to the surface, that is, for $(00 l)$ crystals, but these cases may be treated as $(h 00)$ or $(0 k 0)$ crystals instead.] Then the transformation from the triple-primed axes to the crystal axes is

$$
\begin{aligned}
{\left[\begin{array}{c}
\hat{\mathbf{x}} \\
\hat{\mathbf{y}} \\
\hat{\mathbf{z}}
\end{array}\right]=} & {\left[\begin{array}{ccc}
\frac{h}{\sqrt{h^{2}+k^{2}+l^{2}}} & \frac{-k}{\sqrt{h^{2}+k^{2}}} & \frac{-h l}{\sqrt{h^{2}+k^{2}+l^{2}} \sqrt{h^{2}+k^{2}}} \\
\frac{k}{\sqrt{h^{2}+k^{2}+l^{2}}} & \frac{h}{\sqrt{h^{2}+k^{2}}} & \frac{-k l}{\sqrt{h^{2}+k^{2}+l^{2}} \sqrt{h^{2}+k^{2}}} \\
\frac{l}{\sqrt{h^{2}+k^{2}+l^{2}}} & 0 & \frac{h^{2}+k^{2}}{\sqrt{h^{2}+k^{2}+l^{2}} \sqrt{h^{2}+k^{2}}}
\end{array}\right]\left[\begin{array}{c}
\widehat{\mathbf{x}^{\prime \prime \prime}} \\
\widehat{\mathbf{y}^{\prime \prime \prime}} \\
\widehat{\mathbf{z}^{\prime \prime \prime}}
\end{array}\right] } \\
= & {\left[\begin{array}{lll}
A_{11} & A_{12} & A_{13} \\
A_{21} & A_{22} & A_{23} \\
A_{31} & 0 & A_{33}
\end{array}\right]\left[\begin{array}{c}
\widehat{\mathbf{x}^{\prime \prime \prime}} \\
\widehat{\mathbf{y}^{\prime \prime \prime}} \\
\widehat{\mathbf{z}^{\prime \prime \prime}}
\end{array}\right]=A\left[\begin{array}{c}
\widehat{\mathbf{x}^{\prime \prime \prime}} \\
\widehat{\mathbf{y}} \\
\widehat{\mathbf{z}^{\prime \prime \prime}}
\end{array}\right] . }
\end{aligned}
$$

The crystallographic directions corresponding to the triple-primed axes $\widehat{\mathbf{x}^{\prime \prime \prime}}, \widehat{\mathbf{y}^{\prime \prime \prime}}$, and $\widehat{\mathbf{z}^{\prime \prime \prime}}$ are therefore $[h k l],[\bar{k} h 0]$, and $\left[-h l,-k l, h^{2}+k^{2}\right]$, respectively.

Combining Eqs. (6) and (7), the electric field of the excitation radiation in relation to the crystal axes is

$$
\begin{aligned}
{\left[\begin{array}{l}
E_{x} \\
E_{y} \\
E_{z}
\end{array}\right] } & =\left[\begin{array}{l}
B_{11}+B_{12} \cos \theta+B_{13} \sin \theta \\
B_{21}+B_{22} \cos \theta+B_{23} \sin \theta \\
B_{31}+B_{32} \cos \theta+B_{33} \sin \theta
\end{array}\right] \quad E_{0} \\
& =\left[\begin{array}{lll}
B_{11} & B_{12} & B_{13} \\
B_{21} & B_{22} & B_{23} \\
B_{31} & B_{32} & B_{33}
\end{array}\right]\left[\begin{array}{c}
1 \\
\cos \theta \\
\sin \theta
\end{array}\right] E_{0} .
\end{aligned}
$$

The equations have been written in this way to emphasize the dependence on the azimuthal angle $\theta$. The quantities $B_{i j}$ depend on $h, k, l, \phi^{\prime}$, and $\Phi$, as detailed in Appendix A. For a given crystal face and a fixed incident beam, the quantities $B_{i j}$ are constants.

\section{G. Bulk optical rectification: $d_{14}$}

The second-order nonlinear terms give the bulk polarization field in the crystal in terms of the electric-field components parallel to the crystal axes. The second-order susceptibility may be represented by $\chi_{i j k}^{(2)}(\Omega ;-\omega, \omega)$, as in Eq. (1) of Ref. 18. Here $\Omega$ is the THz frequency (or difference frequency) and $\omega$ is the NIR frequency (or excitation frequency). (The subscript $k$ is conventionally used in this context and should not be confused with the Miller index $k$.) For the $\overline{4} 3 m$ crystals we are considering, the only nonvanishing bulk nonlinear susceptibility term is $d_{14}$. We consider the conditions during the experiment, such as excitation frequency, to be fixed so that $d_{14}$ may be regarded as a constant. Then

$$
\left[\begin{array}{c}
P_{x}^{\text {bulk }} \\
P_{y}^{\text {bulk }} \\
P_{z}^{\text {bulk }}
\end{array}\right]=2 d_{14}\left[\begin{array}{c}
E_{y} E_{z} \\
E_{z} E_{x} \\
E_{x} E_{y}
\end{array}\right] .
$$

In examining the components $E_{i}$ in Eq. (8), it will be seen that the terms that arise in the products $E_{i} E_{j}$ in Eq. (9) will involve constants, $\cos \theta, \sin \theta, \cos \theta \sin \theta$ (which may be re-expressed in terms of $\sin 2 \theta$ ), and $\cos ^{2} \theta$ and $\sin ^{2} \theta$ (both of which may be re-expressed in terms of $\cos 2 \theta$ ). So the bulk polarization may be written as 


$$
\left[\begin{array}{c}
P_{x}^{\text {bulk }} \\
P_{y}^{\text {bulk }} \\
P_{z}^{\text {bulk }}
\end{array}\right]=\left[\begin{array}{l}
C_{11}+C_{12} \cos \theta+C_{13} \sin \theta+C_{14} \cos 2 \theta+C_{15} \sin 2 \theta \\
C_{21}+C_{22} \cos \theta+C_{23} \sin \theta+C_{24} \cos 2 \theta+C_{25} \sin 2 \theta \\
C_{31}+C_{32} \cos \theta+C_{33} \sin \theta+C_{34} \cos 2 \theta+C_{35} \sin 2 \theta
\end{array}\right] .
$$

Here the quantities $C_{i j}$ depend on the terms $h, k, l, \phi^{\prime}, \Phi$, $d_{14}$, and $E_{0}$, as detailed in Appendix A. If these quantities are fixed, which is commonly the case in experiments, the terms $C_{i j}$ are constants.

\section{H. Surface optical rectification: $\gamma^{\prime}$}

In the presence of a surface electric field, the third-order nonlinear terms may give rise to a polarization that is effectively second order. ${ }^{18}$ The third-order susceptibility may be represented by $\chi_{i j k z}^{(3)}(\Omega ;-\omega, \omega, 0)$, as in Eq. (1) of Ref. 29. The final zero corresponds to the $(\mathrm{dc})$ surface field. Here we consider a constant electric field normal to the crystal surface, $\mathbf{F}=F_{0} \widehat{\mathbf{x}^{\prime \prime \prime}}$. For $\overline{4} 3 m$ crystals the polarization components are given by ${ }^{18,19,29}$

$$
\left[\begin{array}{c}
P_{x}^{\text {surf }} \\
P_{y}^{\text {surf }} \\
P_{z}^{\text {surf }}
\end{array}\right]=\left[\begin{array}{c}
\alpha^{\prime} F_{x}+\beta^{\prime} E_{x}+\gamma^{\prime} E_{x}^{2} F_{x} \\
\alpha^{\prime} F_{y}+\beta^{\prime} E_{y}+\gamma^{\prime} E_{y}^{2} F_{y} \\
\alpha^{\prime} F_{z}+\beta^{\prime} E_{z}+\gamma^{\prime} E_{z}^{2} F_{z}
\end{array}\right]
$$

Here $\alpha^{\prime}=3 \chi_{z x x z}\left(E_{x}^{2}+E_{y}^{2}+E_{z}^{2}\right), \beta^{\prime}=6 \chi_{z z x x}\left(E_{x} F_{x}+E_{y} F_{y}+E_{z} F_{z}\right)$, and $\gamma^{\prime}=3\left(\chi_{z z z z}-\chi_{z x x z}-2 \chi_{z z x x}\right)$. We now consider separately the terms involving $\alpha^{\prime}, \beta^{\prime}$, and $\gamma^{\prime}$. Except in the value of $\alpha^{\prime}$, the first term, $\alpha^{\prime} F_{i}$, does not involve the optical field but only the surface field and only has a component normal to the surface. The resulting polarization in the double-primed frame is

$$
\left[\begin{array}{c}
P_{x^{\prime \prime}}^{\text {surf }} \\
P_{y^{\prime \prime}}^{\text {surf }} \\
P_{z^{\prime \prime}}^{\text {surf }}
\end{array}\right]=\left[\begin{array}{l}
1 \\
0 \\
0
\end{array}\right] \alpha^{\prime} F_{0}
$$

Except in the value of $\beta^{\prime}$, the second term, $\beta^{\prime} E_{i}$, does not involve the surface field but only the optical field. The associated polarization in the double-primed frame is

$$
\left[\begin{array}{c}
P_{x^{\prime \prime}}^{\text {surf }} \\
P_{y^{\prime \prime}}^{\text {surf }} \\
P_{z^{\prime \prime}}^{\text {surf }}
\end{array}\right]=\left[\begin{array}{c}
\sin \phi_{\mathrm{NIR}} \cos \Phi \\
\cos \phi_{\mathrm{NIR}} \cos \Phi \\
\sin \Phi
\end{array}\right] \beta^{\prime} E_{0}
$$

So this term involves the incidence angle and the polarization angle but does not involve the azimuthal angle.

We now turn to the third term, $\gamma^{\prime} E_{i}^{2} F_{i}$. Using the components $E_{i}$ in Eq. (8), it will be seen that the terms that arise in $E_{i}^{2}$ may be written in a similar form to Eq. (10):

$$
\left[\begin{array}{c}
P_{x}^{\text {surf }} \\
P_{y}^{\text {surf }} \\
P_{z}^{\text {surf }}
\end{array}\right]=\left[\begin{array}{l}
T_{11}+T_{12} \cos \theta+T_{13} \sin \theta+T_{14} \cos 2 \theta+T_{15} \sin 2 \theta \\
T_{21}+T_{22} \cos \theta+T_{23} \sin \theta+T_{24} \cos 2 \theta+T_{25} \sin 2 \theta \\
T_{31}+T_{32} \cos \theta+T_{33} \sin \theta+T_{34} \cos 2 \theta+T_{35} \sin 2 \theta
\end{array}\right] .
$$

Here the terms $T_{i j}$ depend on the terms $B_{i j}, \gamma^{\prime}, E_{0}$, and $F_{0}$, as detailed in Appendix A. We consider the conditions during the experiment to be fixed such that $\gamma^{\prime}, E_{0}$, and $F_{0}$ may be regarded as constants.

\section{Transforming back to the external crystal coordinate system}

We now reverse the transformations to return to the external frame of reference attached to the crystal surface. Moving from the unprimed to the triple-primed coordinates only involves linear combinations of the previous terms. We consider first the bulk optical rectification terms:

$$
\left[\begin{array}{c}
P_{x^{\prime \prime \prime}}^{\text {bulk }} \\
P_{y^{\prime \prime \prime}}^{\text {bulk }} \\
P_{z^{\prime \prime \prime}}^{\text {bulk }}
\end{array}\right]=A^{-1}\left[\begin{array}{c}
P_{x}^{\text {bulk }} \\
P_{y}^{\text {bulk }} \\
P_{z}^{\text {bulk }}
\end{array}\right]=\left[\begin{array}{l}
D_{11}+D_{12} \cos \theta+D_{13} \sin \theta+D_{14} \cos 2 \theta+D_{15} \sin 2 \theta \\
D_{21}+D_{22} \cos \theta+D_{23} \sin \theta+D_{24} \cos 2 \theta+D_{25} \sin 2 \theta \\
D_{31}+D_{32} \cos \theta+D_{33} \sin \theta+D_{34} \cos 2 \theta+D_{35} \sin 2 \theta
\end{array}\right] .
$$

The quantities $D_{i j}$ depend on $h, k, l, \phi^{\prime}, \Phi, d_{14}$, and $E_{0}$, as detailed in Appendix A.

In a similar way, we can deal with the surface optical rectification effect: 


$$
\left[\begin{array}{c}
P_{x^{\prime \prime \prime}}^{\text {surf }} \\
P_{y^{\prime \prime \prime}}^{\text {surf }} \\
P_{z^{\prime \prime \prime}}^{\text {surf }}
\end{array}\right]=A^{-1}\left[\begin{array}{c}
P_{x}^{\text {surf }} \\
P_{y}^{\text {surf }} \\
P_{z}^{\text {surf }}
\end{array}\right]=\left[\begin{array}{l}
U_{11}+U_{12} \cos \theta+U_{13} \sin \theta+U_{14} \cos 2 \theta+U_{15} \sin 2 \theta \\
U_{21}+U_{22} \cos \theta+U_{23} \sin \theta+U_{24} \cos 2 \theta+U_{25} \sin 2 \theta \\
U_{31}+U_{32} \cos \theta+U_{33} \sin \theta+U_{34} \cos 2 \theta+U_{35} \sin 2 \theta
\end{array}\right] .
$$

The quantities $U_{i j}$ are expressed in detail in Appendix A.

Next, in moving from the triple-primed to the double-primed coordinates, we multiply by $\cos \theta$ and $\sin \theta$ so terms in $\cos 3 \theta$ and $\sin 3 \theta$ emerge:

$$
\begin{aligned}
{\left[\begin{array}{l}
P_{x^{\prime \prime}}^{\text {bulk }} \\
P_{y^{\prime \prime}}^{\text {bulk }} \\
P_{z^{\prime \prime}}^{\text {bulk }}
\end{array}\right] } & =\left[\begin{array}{ccc}
1 & 0 & 0 \\
0 & +\cos \theta+\sin \theta \\
0 & -\sin \theta+\cos \theta
\end{array}\right]\left[\begin{array}{l}
P_{x^{\prime \prime \prime}}^{\text {bulk }} \\
P_{y^{\prime \prime \prime}}^{\text {bulk }} \\
P_{z^{\prime \prime \prime}}^{\text {bulk }}
\end{array}\right] \\
& =\left[\begin{array}{l}
G_{11}+G_{12} \cos \theta+G_{13} \sin \theta+G_{14} \cos 2 \theta+G_{15} \sin 2 \theta+G_{16} \cos 3 \theta+G_{17} \sin 3 \theta \\
G_{21}+G_{22} \cos \theta+G_{23} \sin \theta+G_{24} \cos 2 \theta+G_{25} \sin 2 \theta+G_{26} \cos 3 \theta+G_{27} \sin 3 \theta \\
G_{31}+G_{32} \cos \theta+G_{33} \sin \theta+G_{34} \cos 2 \theta+G_{35} \sin 2 \theta+G_{36} \cos 3 \theta+G_{37} \sin 3 \theta
\end{array}\right] .
\end{aligned}
$$

The quantities $G_{i j}$ may be expressed in terms of $h, k, l, \phi^{\prime}, \Phi, d_{14}$, and $E_{0}$, as detailed in Appendix A.

Likewise, for the surface effect,

$$
\left[\begin{array}{c}
P_{x^{\prime \prime}}^{\text {surf }} \\
P_{y^{\prime \prime}}^{\text {surf }} \\
P_{z^{\prime \prime}}^{\text {surf }}
\end{array}\right]=\left[\begin{array}{l}
V_{11}+V_{12} \cos \theta+V_{13} \sin \theta+V_{14} \cos 2 \theta+V_{15} \sin 2 \theta+V_{16} \cos 3 \theta+V_{17} \sin 3 \theta \\
V_{21}+V_{22} \cos \theta+V_{23} \sin \theta+V_{24} \cos 2 \theta+V_{25} \sin 2 \theta+V_{26} \cos 3 \theta+V_{27} \sin 3 \theta \\
V_{31}+V_{32} \cos \theta+V_{33} \sin \theta+V_{34} \cos 2 \theta+V_{35} \sin 2 \theta+V_{36} \cos 3 \theta+V_{37} \sin 3 \theta
\end{array}\right] .
$$

The quantities $V_{i j}$ may be expressed in terms of $h, k, l, \phi^{\prime}$, $\Phi, \gamma^{\prime}, F_{0}$, and $E_{0}$, as detailed in Appendix A.

We emphasize the significance of these final two results. They demonstrate that, regardless of the crystallographic orientation, the angle of incidence or the angle of polarization, the expressions that arise consist of linear combinations of terms of $\cos n \theta$ and $\sin n \theta$, up to $n=3$ at the most. This observation holds for both the bulk and surface-field optical rectification effects.

\section{J. Detected terahertz field}

We now assume that the $\mathrm{THz}$ field, in the far-field approximation, is directly proportional to the polarization field. Then

$$
\left[\begin{array}{c}
E_{x^{\prime \prime}}^{\mathrm{THz}} \\
E_{y^{\prime \prime}}^{\mathrm{THz}} \\
E_{z^{\prime \prime}}^{\mathrm{THz}}
\end{array}\right]=\left[\begin{array}{c}
P_{x^{\prime \prime}}^{\text {bulk }}+P_{x^{\prime \prime}}^{\text {surf }} \\
P_{y^{\prime \prime}}^{\text {bulk }}+P_{y^{\prime \prime}}^{\text {surf }} \\
P_{z^{\prime \prime}}^{\text {bulk }}+P_{z^{\prime \prime}}^{\text {surf }}
\end{array}\right] Z_{0}
$$

where $Z_{0}$ is a proportionality factor that will depend on experimental parameters such as the distance of the detector from the crystal. We now introduce a coordinate system to refer to the detector. The line running from the point that the excitation beam strikes the crystal to the detector defines the direction $\widehat{\mathbf{x}^{\prime \prime \prime \prime}}$. The angle that this line makes with the $\widehat{\mathbf{z}^{\prime \prime}}$ axis will be called $\xi$. We take $\widehat{\mathbf{y}^{\prime \prime \prime \prime}}$ to lie in the $\widehat{\mathbf{x}^{\prime \prime}}-\widehat{\mathbf{y}^{\prime \prime}}$ plane. Then, in the detector coordinates,

$$
\begin{aligned}
{\left[\begin{array}{c}
E_{x^{\prime \prime \prime \prime}}^{\mathrm{THz}} \\
E_{y^{\prime \prime \prime \prime}}^{\mathrm{THz}} \\
E_{z^{\prime \prime \prime \prime}}^{\mathrm{THz}}
\end{array}\right]=} & {\left[\begin{array}{ccc}
+\sin \xi \cos \phi_{\mathrm{THz}} & -\sin \xi \sin \phi_{\mathrm{THz}}+\cos \xi \\
+\sin \phi_{\mathrm{THz}} & +\cos \phi_{\mathrm{THz}} & 0 \\
-\cos \xi \cos \phi_{\mathrm{THz}} & -\cos \xi \sin \phi_{\mathrm{THz}} & +\sin \xi
\end{array}\right] } \\
& \times\left[\begin{array}{c}
E_{x^{\prime \prime}}^{\mathrm{THz}} \\
E_{y^{\prime \prime}}^{\mathrm{THz}} \\
E_{z^{\prime \prime}}^{\mathrm{THz}}
\end{array}\right] .
\end{aligned}
$$

Here $\phi_{\mathrm{THz}}$ is the angle of refraction in the crystal, related to the angle of reflection, $\phi^{\prime \prime \prime \prime}$, which is measured in the same sense as the angle of incidence, by

$$
n_{\text {air,THz }} \sin \phi^{\prime \prime \prime \prime}=n_{\text {crystal,THz }} \sin \phi_{\mathrm{THz}},
$$

where $n_{\text {air,THz }}$ is the refractive index of the ambient atmosphere at the $\mathrm{THz}$ photon frequency and $n_{\text {crystal, } \mathrm{THz}}$ is the index of the crystal at this photon frequency. We use the term "angle of reflection" for convenience in referring to $\phi^{\prime \prime \prime \prime}$, but note that the detector may be placed anywhere so $\phi^{\prime \prime \prime \prime}$ need bear no particular relation to the angle of incidence $\phi^{\prime}$. We also reiterate that, in general, $n_{\text {crystal, THz }}$ may be different to

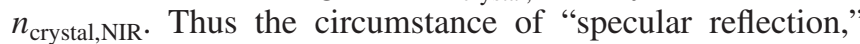
that is, $\phi^{\prime \prime \prime \prime}=\phi^{\prime}$, does not imply that within the crystal $\phi_{\mathrm{NIR}}=\phi_{\mathrm{THz}}$.

In typical experiments, the detector lies in the reflection plane $\left(\xi=90^{\circ}\right)$ and detects either the $\mathrm{THz}$ signal polarized in 
that plane, $E_{p}^{\mathrm{THz}}$, or in the direction perpendicular to this, $E_{s}^{\mathrm{THz}}$. In this case,

$$
\left[\begin{array}{c}
E_{p}^{\mathrm{THz}} \\
E_{s}^{\mathrm{THz}}
\end{array}\right]=\left[\begin{array}{c}
E_{y^{\prime \prime \prime \prime}}^{\mathrm{THz}} \\
E_{z^{\prime \prime \prime \prime}}^{\mathrm{THz}}
\end{array}\right]=\left[\begin{array}{c}
\sin \phi_{\mathrm{THz}} E_{x^{\prime \prime}}^{\mathrm{THz}}+\cos \phi_{\mathrm{THz}} E_{y^{\prime \prime}}^{\mathrm{THz}} \\
E_{z^{\prime \prime}}^{\mathrm{THz}}
\end{array}\right] .
$$

\section{EXPERIMENTAL METHODS}

In the experiments reported here the excitation radiation comprised sub-12-fs pulses of NIR radiation of center wavelength $790 \mathrm{~nm}$ (frequency $379 \mathrm{THz}$, photon energy $1.57 \mathrm{eV}$ ) from a mode-locked Ti:sapphire laser. The laser beam was directed horizontally and was polarized horizontally to the right when looking down the beam. In this arrangement, $\widehat{\mathbf{x}^{\prime}}$ points along the beam back into the laser, $\widehat{\mathbf{y}^{\prime}}$ points to the right when looking down the beam, and $\widehat{\mathbf{z}^{\prime}}$ points up.

A transmission geometry was employed. The excitation beam was perpendicular to the crystal face and the $\mathrm{THz}$ field was detected in the straight-through direction. In this arrangement, transient currents, presumed to be normal to the crystal surface, do not contribute to the $\mathrm{THz}$ signal detected.

So $\mathbf{y}^{\prime \prime}\left(=\widehat{\mathbf{y}^{\prime}}\right.$ in this experiment $)$ ran horizontally along the crystal surface and $\widehat{\mathbf{z}^{\prime \prime}}\left(=\widehat{\mathbf{z}^{\prime}}\right.$ in this experiment) ran upwards along the crystal surface. The polarization angle $\Phi=0$. The azimuthal angle is measured clockwise when facing the crystal, the vertical direction corresponding to $\theta=0$. Appendix B presents the equations of Appendix A for the specific conditions of the experiments we report here: $\phi=0, \Phi=0$, and $(h k l)=(11 N)$.

We now identify the crystal directions parallel to the pump electric field as the azimuthal angle $\theta$ varies. For $\theta$ $=0$, the pump electric field is in the direction of the $\mathbf{y}^{\prime \prime \prime}$ axis, or the $[\bar{k} h 0]$ direction. For $\theta=90^{\circ}$, the pump electric field is in the direction of the $\mathbf{z}^{\prime \prime \prime}$ axis, or the $\left[-h l,-k l, h^{2}+k^{2}\right]$ direction; for $\theta=180^{\circ}$, the $[k \bar{h} 0]$ direction; for $\theta=270^{\circ}$, the $\left[h l, k l,-\left(h^{2}+k^{2}\right)\right]$ direction. For $(11 N)$ crystals these directions are, respectively, [110], $[\bar{N} \bar{N} 2],[1 \overline{1} 0]$, and $[N N \overline{2}]$.

A wire-grid polarizer was used to distinguish the $p$ and $s$ components of the emitted $\mathrm{THz}$ radiation. The $\mathrm{THz}$ radiation was detected electro-optically in free space using a 1-mmthick (110) ZnTe wafer in a conventional $\mathrm{THz}$ time-domain spectroscopy arrangement. The $\mathrm{THz}$ field in the detector crystal is proportional to differential output of a pair of photodiodes which is measured in volts. So in this arrangement

$$
\left[\begin{array}{c}
E_{p}^{\mathrm{THz}} \\
E_{s}^{\mathrm{THz}}
\end{array}\right]=\left[\begin{array}{c}
P_{y^{\prime \prime}}^{\text {bulk }}+P_{y^{\prime \prime}}^{\text {surf }} \\
P_{z^{\prime \prime}}^{\text {bulk }}+P_{z^{\prime \prime}}^{\text {surf }}
\end{array}\right] Z,
$$

where $Z$ is a conversion factor that depends on the experimental arrangement but does not change throughout the experiments.

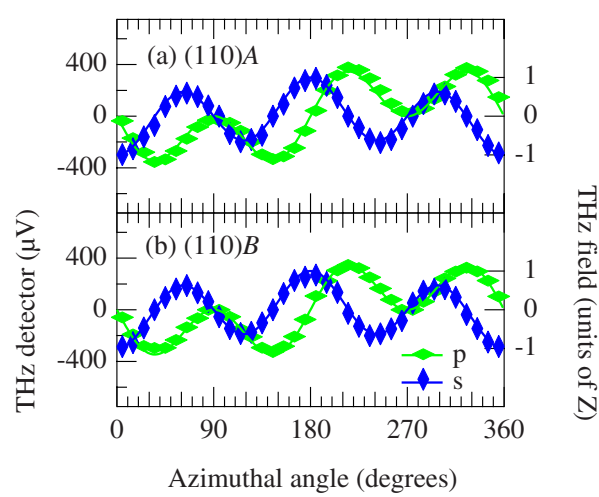

FIG. 2. (Color online) $\mathrm{THz}$ emission from (a) (110)A and (b) (110) $B$ faces of GaAs. The symbols are the experimental data and refer the left axis. The full lines are the theory for bulk optical rectification from a (110) face and relate to the right axis. The axis ranges are the same for both parts (a) and (b).

\section{RESULTS AND DISCUSSION}

We begin with the simple case of a GaAs (110) crystal. For this crystal face, the theory developed in Sec. II predicts no contribution from the surface field to the OR. Data for both the $A$ and $B$ faces are given in Fig. 2. All four sets of data in Fig. 2 ( $A$ and $B$ faces, $p$ and $s$ components) are plotted on the same $\mathrm{THz}$ detector $(\mu \mathrm{V})$ axis without any scaling. Likewise, the calculations are all plotted on the same axis relative to $Z$ without any scaling. So there is only a single fitting parameter to match theory with experiment, given in these experiments by setting $Z=1$ to correspond to $300 \mu \mathrm{V}$.

It may be seen from Fig. 2 that the data for both $A$ and $B$ faces (symbols) are well accounted for by the calculation involving bulk OR alone (full lines). Possible differences of the faces, for example, due to different surface reconstructions do not lead to any discernible difference in the $\mathrm{THz}$ field detected.

We next consider the case of GaAs $(11 N) B$ crystal faces with $N=2,3,4$, and 5 . The $\mathrm{THz}$ field detected as a function of azimuthal angle is given in Fig. 3. Several observations may be made from the experimental data, shown by the horizontally and vertically oriented lozenges. First, the $p$-polarized component is dominated by a (negative) $\sin 3 \theta$ term and the $s$-polarized component is dominated by a (negative) $\cos 3 \theta$ term in all cases. Second, more subtly, the $p$-polarized components exhibit one major maximum and two minor maxima, whereas the $s$-polarized components exhibit two major maxima and one minor maximum. This suggests an additional $\sin \theta$ and an additional $\cos \theta$ term, respectively, are involved. Third, the $\mathrm{THz}$ field strength decreases as the third Miller index $N$ increases.

The experimental data in Fig. 3 will now be compared with the calculation for $\mathrm{THz}$ emission due to bulk OR, which proved to very adequately account for the (110) data as shown in Fig. 2. In the experimental configuration employed here, $\phi^{\prime}=\Phi=0, h=k=1$, and $l=N$. The equations for the polarization components due to bulk OR, given in general form in Appendix A, become in this specific case 


$$
\begin{aligned}
& P_{y^{\prime \prime}}^{\text {bulk }}=\left[3\left(N^{2}-1\right) \sin \theta-3\left(N^{2}+1\right) \sin 3 \theta\right] \frac{d_{14} E_{0}^{2}}{\sqrt{2}\left(N^{2}+2\right)^{3 / 2}}, \\
& P_{z^{\prime \prime}}^{\text {bulk }}=\left[\left(N^{2}-1\right) \cos \theta-3\left(N^{2}+1\right) \cos 3 \theta\right] \frac{d_{14} E_{0}^{2}}{\sqrt{2}\left(N^{2}+2\right)^{3 / 2}} .
\end{aligned}
$$

These expressions, with $d_{14} E_{0}^{2}$ set to 1 , are plotted as dashed lines in Fig. 3 for $N=2,3,4$, and 5. It may be seen that the theory broadly reproduces the features of the experimental observations. In Eqs. (25) and (26), the coefficients of $\sin 3 \theta$ and $\cos 3 \theta$ are the same, but the coefficient of $\sin \theta$ is three times that of the coefficient of $\cos \theta$, leading to a stronger divergence from $3 \theta$ behavior for the $p$-polarized radiation than for the $s$-polarized radiation. The observation that the $\mathrm{THz}$ radiated lessens as $N$ increases may be physically understood as the $(11 N)$ face approaching the (001) face, for which, in the transmission geometry as employed here, $P_{y^{\prime \prime}}^{\text {bulk }}=P_{z^{\prime \prime}}^{\text {bulk }}=0$.

While the overall features displayed by the experimental data (symbols) in Fig. 3 are reproduced by the bulk OR theory (dashed lines), better agreement may be obtained by including in the theory a surface-field contribution to the OR. The fact that the data in Fig. 3 are not offset from zero indicates that the $\alpha^{\prime}$ and $\beta^{\prime}$ surface-field terms make negligible contribution to the OR. These terms will not be considered further. We now present the expressions for $P_{y^{\prime \prime}}$ and $P_{z^{\prime \prime}}$ that result when both the surface-field and the bulk contributions to the OR are taken into account in the case of a (11N) face in transmission geometry. These are

$$
\begin{aligned}
P_{y^{\prime \prime}}^{\text {bulk }+ \text { surf }}= & \left\{3\left[2\left(N^{2}-1\right)\left(N^{2}+2\right)^{1 / 2} d_{14}-N\left(N^{2}-1\right) \gamma^{\prime} F_{0}\right] \sin \theta\right. \\
& -\left[6\left(N^{2}+1\right)\left(N^{2}+2\right)^{1 / 2} d_{14}\right. \\
& \left.\left.+N\left(N^{2}+5\right) \gamma^{\prime} F_{0}\right] \sin 3 \theta\right\} \times \frac{E_{0}^{2}}{2 \sqrt{2}\left(N^{2}+2\right)^{2}}, \quad(27) \\
P_{z^{\prime \prime}}^{\text {bulk }+ \text { surf }}= & \left\{\left[2\left(N^{2}-1\right)\left(N^{2}+2\right)^{1 / 2} d_{14}-N\left(N^{2}-1\right) \gamma^{\prime} F_{0}\right] \cos \theta\right. \\
& -\left[6\left(N^{2}+1\right)\left(N^{2}+2\right)^{1 / 2} d_{14}\right. \\
& \left.\left.+N\left(N^{2}+5\right) \gamma^{\prime} F_{0}\right] \cos 3 \theta\right\} \times \frac{E_{0}^{2}}{2 \sqrt{2}\left(N^{2}+2\right)^{2}} .
\end{aligned}
$$

As for bulk OR in Eqs. (25) and (26), the coefficients of $\sin 3 \theta$ and $\cos 3 \theta$ are the same in Eqs. (27) and (28), and the coefficient of $\sin \theta$ is three times that of the coefficient of $\cos \theta$, leading to a stronger divergence from $3 \theta$ behavior for the $p$-polarized radiation than for the $s$-polarized radiation. It may be noted that for $N=0$ all the terms in Eqs. (27) and (28) involving the surface field $F_{0}$ vanish. Thus, even if there is a surface field at a (110) $A$ or (110) $B$ crystal face it will not contribute to $\mathrm{THz}$ emission. This explains why the (110) data in Fig. 2 is accounted for by the bulk OR alone. No conclusion regarding the presence or not of a surface field can be made from that data.

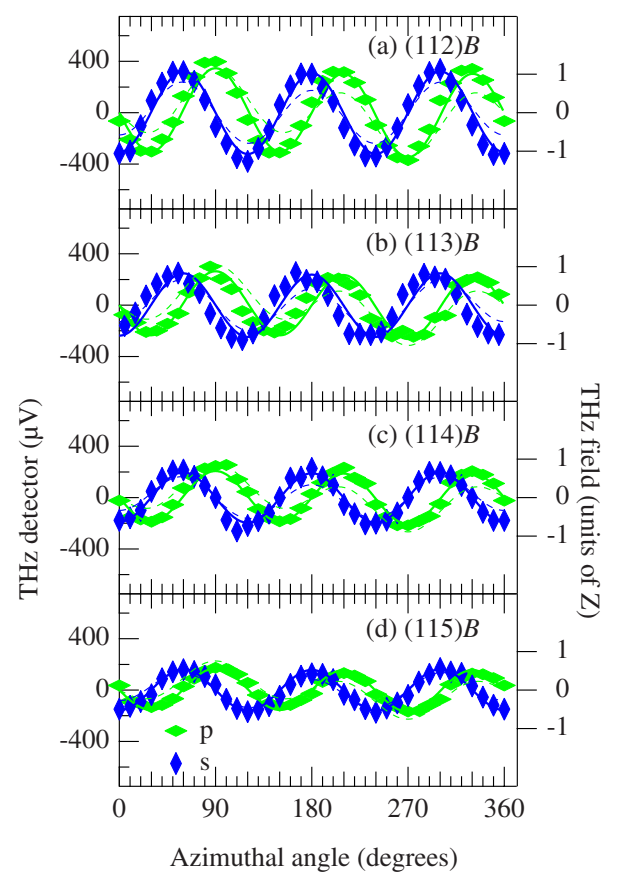

FIG. 3. (Color online) Experimental data (symbols) for $\mathrm{THz}$ emission from $(11 N) B$ faces of GaAs. The dashed lines are the calculations for bulk optical rectification alone (with $d_{14} E_{0}^{2}=1$ ). The full lines are the calculations for combined bulk $\left(d_{14} E_{0}^{2}=1\right)$ and surface $\left(\gamma^{\prime} F_{0}=1.9 d_{14}\right)$ optical rectification.

In comparing the calculation to the experimental data of Fig. 3 we first set the bulk parameter to be the same as was found for the (110) case; that is, setting $d_{14} E_{0}^{2}=1$ means $Z$ $=1$ in our experiments corresponds to $300 \mu \mathrm{V}$, as mentioned earlier. Then we vary the surface parameter $\gamma^{\prime} F_{0}$. We find the ratio $\gamma^{\prime} F_{0}=(+1.9 \pm 0.2) d_{14}$ fits the data well. The full lines in Fig. 3 are shown for $\gamma^{\prime} F_{0}=1.9 d_{14}$. We stated earlier that only a single fitting parameter, the bulk term, was needed to fit all four sets of experimental data for the (110) faces in Fig. 2. Given the bulk term, only a single fitting parameter, the surface term, is now needed to fit all eight sets of experimental data [both $p$ and $s$ polarizations for (112) $B,(113) B,(114) B$, and (115) $B$ faces] in Fig. 3.

Finally, we turn to GaAs $(11 N) A$ crystal faces. The $\mathrm{THz}$ field detected as a function of azimuthal angle for (112)A, (113) $A$, (114) $A$, and (115) $A$ GaAs crystals is given by the symbols in Fig. 4. In comparing the data from the $A$ faces (Fig. 4) and $B$ faces (Fig. 3), it may be seen that the $s$-polarized components are similar, with a clear (negative) $\sin 3 \theta$ behavior and the suggestion of an additional small $\sin \theta$ term. The $p$-polarized components are quite different. The dashed lines in Fig. 4 (identical to the dashed lines in Fig. 3) give the theory for the bulk-only OR, which does not fully account for the observations. We now adopt the same bulk term employed in the $(110)$ and $(11 N) B$ cases $\left(d_{14} E_{0}^{2}\right.$ $=1)$ and vary the surface term $\left(\gamma^{\prime} F_{0}\right)$. We find the ratio $\gamma^{\prime} F_{0}=(-1.6 \pm 0.2) d_{14}$ fits the data. The calculations for $\gamma^{\prime} F_{0}=-1.6 d_{14}$ are given in Fig. 4 by the full lines. In this figure, the same ratio of $\gamma^{\prime} F_{0}$ to $d_{14}$ is employed for each panel, corresponding to the same surface field in each sample. Variations in the spectra, such as the smoothing of 


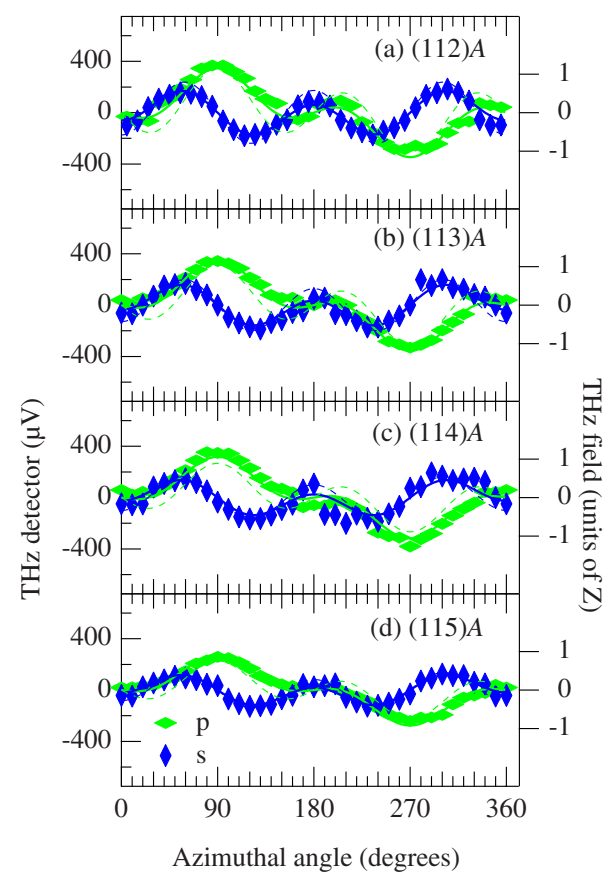

FIG. 4. (Color online) Experimental data (symbols) for $\mathrm{THz}$ emission from $(11 N) A$ faces of GaAs. The dashed lines are the calculations for bulk optical rectification alone (with $d_{14} E_{0}^{2}=1$ ). The full lines are the calculations for combined bulk $\left(d_{14} E_{0}^{2}=1\right)$ and surface $\left(\gamma^{\prime} F_{0}=-1.6 d_{14}\right)$ optical rectification.

the central "wiggle" in the $p$ component as $N$ increases, arise naturally from the Eqs. (27) and (28) through the terms involving $N$. There is no evidence from these data that $F_{0}$ varies between the samples.

\section{CONCLUSION}

General expressions for bulk and surface contributions to OR for planes of arbitrary orientation in zinc-blende crystals have been developed and are set out in detail in Appendix A. The equations are completely general with respect to the angle of incidence and of polarization of the pump beam and with respect to the location of the detector of the $\mathrm{THz}$ radiation. To illustrate the utility of these general results in a specific application, the particular equations relating to $(11 \mathrm{~N})$ crystals are set out in Appendix B. From symmetry considerations alone, it is seen that in a (110) crystal a surface field does not contribute to $\mathrm{THz}$ emission in the transmission geometry. In our experiments we therefore utilize (110) GaAs crystals to determine the contribution of GaAs bulk OR alone without the complication of surface OR. As expected from the theory, the emission of $\mathrm{THz}$ radiation from $\mathrm{GaAs}$ (110) $A$ and (110) $B$ faces is measured to be the same. The azimuthal-angle dependence is fitted using a single parameter proportional to the nonlinear optical constant $d_{14}$. Using the same experimental arrangement and the value of the bulk parameter obtained from the (110) measurements, we then determined the surface-field contributions to the $\mathrm{THz}$ emission from GaAs $(11 N) A$ and $(11 N) B$ faces. Within the set (112) $A$, (113) $A,(114) A$, and (115)A we find the same surface field, which may be expressed by $F_{0_{A}}$
$=(-1.6 \pm 0.2) d_{14} / \gamma^{\prime}$, where $\gamma^{\prime}$ is a nonlinear optical constant. Within the set (112) $B,(113) B,(114) B$, and (115) $B$ we find the same surface field, $F_{0_{B}}=(1.9 \pm 0.2) d_{14} / \gamma^{\prime}$. Thus the surface fields on the Ga-rich and As-rich faces are, within experimental error, of the same strength, but point away from and into the surfaces, respectively. Such conclusions about the surface fields that are not accessible by other methods.

\section{ACKNOWLEDGMENTS}

This work was supported by the Australian Research Council and by the University of Wollongong. We thank M. Henini for provision of the samples.

APPENDIX A: GENERAL CASE

$$
\begin{gathered}
m=\sqrt{h^{2}+k^{2}+l^{2},} \\
n=\sqrt{h^{2}+k^{2}}, \\
s=\sin \phi_{\mathrm{NIR}} \cos \Phi, \\
c=\cos \phi_{\mathrm{NIR}} \cos \Phi, \\
t=\sin \Phi, \\
d=d_{14} E_{0}^{2}, \\
A_{33}=\frac{n}{m}, \\
A_{32} F_{0} E_{0}^{2}, \\
A_{23}=-\frac{k}{m n}, \\
A_{11}=\frac{h}{m}, \\
A_{21}=\frac{k}{m}, \\
A_{12}=-\frac{k}{n}, \\
A_{13}, \\
A_{22},
\end{gathered}
$$




$$
\begin{aligned}
& B_{11}=\frac{h s}{m}, \\
& B_{12}=-\frac{k c}{n}-\frac{h l t}{m n}, \\
& B_{13}=-\frac{h l c}{m n}+\frac{k t}{n} \\
& B_{21}=\frac{k s}{m}, \\
& B_{22}=\frac{h c}{n}-\frac{k l t}{m n}, \\
& B_{23}=-\frac{k l c}{m n}-\frac{h t}{n}, \\
& B_{31}=\frac{l s}{m}, \\
& B_{32}=\frac{n t}{m}, \\
& B_{33}=\frac{n c}{m}, \\
& C_{11} / d=\frac{k l}{m^{2}}\left(2 s^{2}-c^{2}-t^{2}\right), \\
& C_{12} / d=\frac{2 h l}{m n} s c+\frac{2 k\left(h^{2}+k^{2}-l^{2}\right)}{m^{2} n} s t, \\
& C_{13} / d=\frac{2 k\left(h^{2}+k^{2}-l^{2}\right)}{m^{2} n} s c-\frac{2 h l}{m n} s t, \\
& C_{14} / d=\frac{k l}{m^{2}}\left(c^{2}-t^{2}\right)+\frac{2 h}{m} c t, \\
& C_{15} / d=\frac{h}{m}\left(c^{2}-t^{2}\right)-\frac{2 k l}{m^{2}} c t, \\
& C_{21} / d=\frac{h l}{m^{2}}\left(2 s^{2}-c^{2}-t^{2}\right), \\
& C_{22} / d=-\frac{2 k l}{m n} s c+\frac{2 h\left(h^{2}+k^{2}-l^{2}\right)}{m^{2} n} s t, \\
& C_{23} / d=\frac{2 h\left(h^{2}+k^{2}-l^{2}\right)}{m^{2} n} s c+\frac{2 k l}{m n} s t, \\
& C_{24} / d=\frac{h l}{m^{2}}\left(c^{2}-t^{2}\right)-\frac{2 k}{m} c t,
\end{aligned}
$$

$$
\begin{gathered}
D_{11} / d=\frac{3 h k l}{m^{3}}\left(2 s^{2}-c^{2}-t^{2}\right), \\
D_{12} / d=\frac{4 l\left(h^{2}-k^{2}\right)}{m^{2} n} s c+\frac{4 h k\left(h^{2}+k^{2}-2 l^{2}\right)}{m^{3} n} s t, \\
D_{13} / d=\frac{4 h k\left(h^{2}+k^{2}-2 l^{2}\right)}{m^{3} n} s c-\frac{4 l\left(h^{2}-k^{2}\right)}{m^{2} n} s t, \\
D_{14} / d=\frac{h k l\left(h^{2}+k^{2}-2 l^{2}\right)}{m^{3} n^{2}}\left(c^{2}-t^{2}\right) \\
+\frac{2\left(h^{2}-k^{2}\right)\left(h^{2}+k^{2}-l^{2}\right)}{m^{2} n^{2}} c t, \\
D_{15} / d=\frac{\left(h^{2}-k^{2}\right)\left(h^{2}+k^{2}-l^{2}\right)}{m^{2} n^{2}}\left(c^{2}-t^{2}\right) \\
-\frac{2 h k l\left(h^{2}+k^{2}-2 l^{2}\right)}{m^{3} n^{2}} c t, \\
D_{24} / d=\frac{l\left(h^{2}-k^{2}\right)}{m^{2} n}\left(c^{2}-t^{2}\right)-\frac{4 h k}{m n} c t, \\
D_{23} / d=\frac{2\left(h^{2}-k^{2}\right)\left(h^{2}+k^{2}-l^{2}\right)}{m^{2} n^{2}} s c+\frac{4 h k l}{m n^{2}} s t, \\
D_{22} / d=-\frac{4 h k l}{m n^{2} n} s c+\frac{2\left(h^{2}-k^{2}\right)\left(h^{2}+k^{2}-l^{2}\right)}{m^{2} n^{2}} s t, \\
\left.m^{2}-t^{2}\right),
\end{gathered}
$$




$$
\begin{aligned}
& D_{25} / d=-\frac{2 h k}{m n}\left(c^{2}-t^{2}\right)-\frac{2 l\left(h^{2}-k^{2}\right)}{m^{2} n} c t, \\
& D_{31} / d=\frac{h k\left(h^{2}+k^{2}-2 l^{2}\right)}{m^{3} n}\left(2 s^{2}-c^{2}-t^{2}\right), \\
& D_{32} / d=\frac{2\left(h^{2}-k^{2}\right)\left(h^{2}+k^{2}-l^{2}\right)}{m^{2} n^{2}} s c-\frac{4 h k l\left(2 h^{2}+2 k^{2}-l^{2}\right)}{m^{3} n^{2}} s t, \\
& D_{33} / d=-\frac{4 h k l\left(2 h^{2}+2 k^{2}-l^{2}\right)}{m^{3} n^{2}} s c-\frac{2\left(h^{2}-k^{2}\right)\left(h^{2}+k^{2}-l^{2}\right)}{m^{2} n^{2}} s t, \\
& D_{34} / d=-\frac{h k\left(h^{2}+k^{2}+4 l^{2}\right)}{m^{3} n}\left(c^{2}-t^{2}\right)-\frac{4 l\left(h^{2}-k^{2}\right)}{m^{2} n} c t, \\
& D_{35} / d=-\frac{2 l\left(h^{2}-k^{2}\right)}{m^{2} n}\left(c^{2}-t^{2}\right)+\frac{2 h k\left(h^{2}+k^{2}+4 l^{2}\right)}{m^{3} n} c t, \\
& G_{11} / d=\frac{3 h k l}{m^{3}}\left(2 s^{2}-c^{2}-t^{2}\right), \\
& G_{12} / d=\frac{4 l\left(h^{2}-k^{2}\right)}{m^{2} n} s c+\frac{4 h k\left(h^{2}+k^{2}-2 l^{2}\right)}{m^{3} n} s t, \\
& G_{13} / d=\frac{4 h k\left(h^{2}+k^{2}-2 l^{2}\right)}{m^{3} n} s c-\frac{4 l\left(h^{2}-k^{2}\right)}{m^{2} n} s t, \\
& G_{14} / d=\frac{h k l\left(h^{2}+k^{2}-2 l^{2}\right)}{m^{3} n^{2}}\left(c^{2}-t^{2}\right) \\
& +\frac{2\left(h^{2}-k^{2}\right)\left(h^{2}+k^{2}-l^{2}\right)}{m^{2} n^{2}} c t, \\
& G_{15} / d=\frac{\left(h^{2}-k^{2}\right)\left(h^{2}+k^{2}-l^{2}\right)}{m^{2} n^{2}}\left(c^{2}-t^{2}\right) \\
& -\frac{2 h k l\left(h^{2}+k^{2}-2 l^{2}\right)}{m^{3} n^{2}} c t, \\
& G_{16} / d=0, \\
& G_{17} / d=0 \text {, } \\
& G_{21} / d=-\frac{6 h k l}{m^{3}} s c,
\end{aligned}
$$

$$
\begin{gathered}
G_{31} / d=-\frac{6 h k l}{m^{3}} s t, \quad(\mathrm{~A} 70) \\
G_{32} / d=\frac{h k\left(h^{2}+k^{2}-2 l^{2}\right)}{2 m^{3} n}\left(4 s^{2}-c^{2}-3 t^{2}\right)-\frac{l\left(h^{2}-k^{2}\right)}{m^{2} n} c t, \\
G_{33} / d=-\frac{l\left(h^{2}-k^{2}\right)}{2 m^{2} n}\left(4 s^{2}-c^{2}-3 t^{2}\right)-\frac{h k\left(h^{2}+k^{2}-2 l^{2}\right)}{m^{3} n} c t, \\
G_{34} / d=\frac{2\left(h^{2}-k^{2}\right)\left(h^{2}+k^{2}-l^{2}\right)}{m^{2} n^{2}} s c-\frac{2 h k l\left(h^{2}+k^{2}-2 l^{2}\right)}{m^{3} n^{2}} s t, \\
G_{35} / d=-\frac{2 h k l\left(h^{2}+k^{2}-2 l^{2}\right)}{m^{3} n^{2}} s c-\frac{2\left(h^{2}-k^{2}\right)\left(h^{2}+k^{2}-l^{2}\right)}{m^{2} n^{2}} s t,
\end{gathered}
$$$$
G_{23} / d=\frac{h k\left(h^{2}+k^{2}-2 l^{2}\right)}{2 m^{3} n}\left(4 s^{2}-3 c^{2}-t^{2}\right)+\frac{l\left(h^{2}-k^{2}\right)}{m^{2} n} c t,
$$$$
G_{24} / d=\frac{2 h k l\left(h^{2}+k^{2}-2 l^{2}\right)}{m^{3} n^{2}} s c+\frac{2\left(h^{2}-k^{2}\right)\left(h^{2}+k^{2}-l^{2}\right)}{m^{2} n^{2}} s t,
$$

$$
G_{25} / d=\frac{2\left(h^{2}-k^{2}\right)\left(h^{2}+k^{2}-l^{2}\right)}{m^{2} n^{2}} s c-\frac{2 h k l\left(h^{2}+k^{2}-2 l^{2}\right)}{m^{3} n^{2}} s t,
$$$$
G_{26} / d=\frac{3 l\left(h^{2}-k^{2}\right)}{2 m^{2} n}\left(c^{2}-t^{2}\right)-\frac{3 h k\left(h^{2}+k^{2}+2 l^{2}\right)}{m^{3} n} c t,
$$$$
G_{27} / d=-\frac{3 h k\left(h^{2}+k^{2}+2 l^{2}\right)}{2 m^{3} n}\left(c^{2}-t^{2}\right)-\frac{3 l\left(h^{2}-k^{2}\right)}{m^{2} n} c t,
$$

$$
G_{36} / d=-\frac{3 h k\left(h^{2}+k^{2}+2 l^{2}\right)}{2 m^{3} n}\left(c^{2}-t^{2}\right)-\frac{3 l\left(h^{2}-k^{2}\right)}{m^{2} n} c t,
$$

$$
G_{37} / d=-\frac{3 l\left(h^{2}-k^{2}\right)}{2 m^{2} n}\left(c^{2}-t^{2}\right)+\frac{3 h k\left(h^{2}+k^{2}+2 l^{2}\right)}{m^{3} n} c t,
$$

$$
G_{22} / d=\frac{l\left(h^{2}-k^{2}\right)}{2 m^{2} n}\left(4 s^{2}-3 c^{2}-t^{2}\right)-\frac{h k\left(h^{2}+k^{2}-2 l^{2}\right)}{m^{3} n} c t,
$$

$$
T_{11} / g=\frac{h^{3}}{m^{3}} s^{2}+\frac{h\left(k^{2}+l^{2}\right)}{2 m^{3}}\left(c^{2}+t^{2}\right),
$$




$$
\begin{aligned}
& T_{12} / g=-\frac{2 h^{2} k}{m^{2} n} s c-\frac{2 h^{3} l}{m^{3} n} s t, \\
& T_{13} / g=-\frac{2 h^{3} l}{m^{3} n} s c+\frac{2 h^{2} k}{m^{2} n} s t, \\
& T_{14} / g=\frac{h\left(h^{2} k^{2}+k^{4}+k^{2} l^{2}-h^{2} l^{2}\right)}{2 m^{3} n^{2}}\left(c^{2}-t^{2}\right)+\frac{2 h^{2} k l}{m^{2} n^{2}} c t, \\
& T_{15} / g=\frac{h^{2} k l}{m^{2} n^{2}}\left(c^{2}-t^{2}\right)-\frac{h\left(h^{2} k^{2}+k^{4}+k^{2} l^{2}-h^{2} l^{2}\right)}{m^{3} n^{2}} c t, \\
& T_{21} / g=\frac{k^{3}}{m^{3}} s^{2}+\frac{k\left(h^{2}+l^{2}\right)}{2 m^{3}}\left(c^{2}+t^{2}\right), \\
& T_{22} / g=\frac{2 h k^{2}}{m^{2} n} s c-\frac{2 k^{3} l}{m^{3} n} s t, \\
& T_{23} / g=-\frac{2 k^{3} l}{m^{3} n} s c-\frac{2 h k^{2}}{m^{2} n} s t, \\
& T_{24} / g=\frac{k\left(h^{4}+h^{2} k^{2}+h^{2} l^{2}-k^{2} l^{2}\right)}{2 m^{3} n^{2}}\left(c^{2}-t^{2}\right)-\frac{2 h k^{2} l}{m^{2} n^{2}} c t, \\
& T_{25} / g=-\frac{h k^{2} l}{m^{2} n^{2}}\left(c^{2}-t^{2}\right)-\frac{k\left(h^{4}+h^{2} k^{2}+h^{2} l^{2}-k^{2} l^{2}\right)}{m^{3} n^{2}} c t, \\
& T_{31} / g=\frac{l^{3}}{m^{3}} s^{2}+\frac{l\left(h^{2}+k^{2}\right)}{2 m^{3}}\left(c^{2}+t^{2}\right), \\
& T_{32} / g=\frac{2 l^{2} n}{m^{3}} s t, \\
& T_{33} / g=\frac{2 l^{2} n}{m^{3}} s c, \\
& T_{34} / g=-\frac{\left(h^{2}+k^{2}\right) l}{2 m^{3}}\left(c^{2}-t^{2}\right), \\
& T_{35} / g=\frac{l\left(h^{2}+k^{2}\right)}{m^{3}} c t, \\
& U_{11} / g=\frac{\left(h^{4}+k^{4}+l^{4}\right)}{m^{4}} s^{2}+\frac{\left(h^{2} k^{2}+h^{2} l^{2}+k^{2} l^{2}\right)}{m^{4}}\left(c^{2}+t^{2}\right), \\
& U_{25} / g=-\frac{2 h^{2} k^{2} l}{m^{2} n^{3}}\left(c^{2}-t^{2}\right)-\frac{h k\left(h^{2}-k^{2}\right)\left(h^{2}+k^{2}+2 l^{2}\right)}{m^{3} n^{3}} c t, \\
& U_{15} / g=\frac{h k l\left(h^{2}-k^{2}\right)}{m^{3} n^{2}}\left(c^{2}-t^{2}\right)-\frac{2\left(h^{4} k^{2}+h^{2} k^{4}-h^{4} l^{2}-k^{4} l^{2}\right)}{m^{4} n^{2}} c t, \\
& U_{21} / g=-\frac{h k\left(h^{2}-k^{2}\right)}{2 m^{3} n}\left(2 s^{2}-c^{2}-t^{2}\right) \\
& U_{22} / g=\frac{4 h^{2} k^{2}}{m^{2} n^{2}} s c+\frac{2 h k l\left(h^{2}-k^{2}\right)}{m^{3} n^{2}} s t, \\
& U_{23} / g=\frac{2 h k l\left(h^{2}-k^{2}\right)}{m^{3} n^{2}} s c-\frac{4 h^{2} k^{2}}{m^{2} n^{2}} s t, \\
& U_{24} / g=\frac{h k\left(h^{2}-k^{2}\right)\left(h^{2}+k^{2}+2 l^{2}\right)}{2 m^{3} n^{3}}\left(c^{2}-t^{2}\right)-\frac{4 h^{2} k^{2} l}{m^{2} n^{3}} c t, \\
& U_{12} / g=-\frac{2 h k\left(h^{2}-k^{2}\right)}{m^{3} n} s c+\frac{2 l\left(h^{2} l^{2}+k^{2} l^{2}-h^{4}-k^{4}\right)}{m^{4} n} s t, \\
& U_{13} / g=\frac{2 l\left(h^{2} l^{2}+k^{2} l^{2}-h^{4}-k^{4}\right)}{m^{4} n} s c+\frac{2 h k\left(h^{2}-k^{2}\right)}{m^{3} n} s t, \\
& U_{14} / g=\frac{\left(h^{4} k^{2}+h^{2} k^{4}-h^{4} l^{2}-k^{4} l^{2}\right)}{m^{4} n^{2}}\left(c^{2}-t^{2}\right)+\frac{2 h k l\left(h^{2}-k^{2}\right)}{m^{3} n^{2}} c t, \\
& U_{31} / g=\frac{l\left(h^{2} l^{2}+k^{2} l^{2}-h^{4}-k^{4}\right)}{2 m^{4} n}\left(2 s^{2}-c^{2}-t^{2}\right), \\
& U_{32} / g=\frac{2 h k l\left(h^{2}-k^{2}\right)}{m^{3} n^{2}} s c+\frac{4 l^{2}\left(h^{4}+h^{2} k^{2}+k^{4}\right)}{m^{4} n^{2}} s t, \\
& U_{33} / g=\frac{4 l^{2}\left(h^{4}+h^{2} k^{2}+k^{4}\right)}{m^{4} n^{2}} s c-\frac{2 h k l}{m^{3} n^{2}}\left(h^{2}-k^{2}\right) s t, \\
& U_{34} g=\frac{l\left(h^{4} l^{2}+k^{4} l^{2}-5 h^{4} k^{2}-5 h^{2} k^{4}-2 h^{2} k^{2} l^{2}-h^{6}-k^{6}\right)}{2 m^{4} n^{3}}\left(c^{2}\right. \\
& \left.-t^{2}\right)-\frac{2 h k l^{2}\left(h^{2}-k^{2}\right)}{m^{3} n^{3}} c t,
\end{aligned}
$$




$$
\begin{aligned}
U_{35} / g= & -\frac{h k l^{2}\left(h^{2}-k^{2}\right)}{m^{3} n^{3}}\left(c^{2}-t^{2}\right) \\
& -\frac{l\left(h^{4} l^{2}+k^{4} l^{2}-5 h^{4} k^{2}-5 h^{2} k^{4}-2 h^{2} k^{2} l^{2}-h^{6}-k^{6}\right)}{m^{4} n^{3}} c t,
\end{aligned}
$$

$$
\begin{aligned}
& V_{11} / g=\frac{\left(h^{4}+k^{4}+l^{4}\right)}{m^{4}} s^{2}+\frac{\left(h^{2} k^{2}+h^{2} l^{2}+k^{2} l^{2}\right)}{m^{4}}\left(c^{2}+t^{2}\right), \\
& V_{12} / g=-\frac{2 h k\left(h^{2}-k^{2}\right)}{m^{3} n} s c+\frac{2 l\left(h^{2} l^{2}+k^{2} l^{2}-h^{4}-k^{4}\right)}{m^{4} n} s t \\
& V_{13} / g=\frac{2 l\left(h^{2} l^{2}+k^{2} l^{2}-h^{4}-k^{4}\right)}{m^{4} n} s c+\frac{2 h k\left(h^{2}-k^{2}\right)}{m^{3} n} s t
\end{aligned}
$$$$
V_{14} / g=\frac{\left(h^{4} k^{2}+h^{2} k^{4}-h^{4} l^{2}-k^{4} l^{2}\right)}{m^{4} n^{2}}\left(c^{2}-t^{2}\right)+\frac{2 h k l\left(h^{2}-k^{2}\right)}{m^{3} n^{2}} c t,
$$

$$
V_{15} / g=\frac{h k l\left(h^{2}-k^{2}\right)}{m^{3} n^{2}}\left(c^{2}-t^{2}\right)-\frac{2\left(h^{4} k^{2}+h^{2} k^{4}-h^{4} l^{2}-k^{4} l^{2}\right)}{m^{4} n^{2}} c t,
$$

$$
\begin{gathered}
V_{16} / g=0 \\
V_{17} / g=0 \\
V_{21} / g=\frac{2\left(h^{2} k^{2}+h^{2} l^{2}+k^{2} l^{2}\right)}{m^{4}} s c \\
V_{22} / g=-\frac{h k\left(h^{2}-k^{2}\right)}{4 m^{3} n}\left(4 s^{2}-3 c^{2}-t^{2}\right) \\
-\frac{l\left(h^{2} l^{2}+k^{2} l^{2}-h^{4}-k^{4}\right)}{2 m^{4} n} c t \\
V_{23} / g=\frac{l\left(h^{2} l^{2}+k^{2} l^{2}-h^{4}-k^{4}\right)}{4 m^{4} n}\left(4 s^{2}-3 c^{2}-t^{2}\right) \\
-\frac{h k\left(h^{2}-k^{2}\right)}{2 m^{3} n} c t
\end{gathered}
$$

$$
V_{24} / g=\frac{2\left(h^{4} k^{2}+h^{2} k^{4}-h^{4} l^{2}-k^{4} l^{2}\right)}{m^{4} n^{2}} s c+\frac{2 h k l\left(h^{2}-k^{2}\right)}{m^{3} n^{2}} s t,
$$

$$
V_{25} / g=\frac{2 h k l\left(h^{2}-k^{2}\right)}{m^{3} n^{2}} s c-\frac{2\left(h^{4} k^{2}+h^{2} k^{4}-h^{4} l^{2}-k^{4} l^{2}\right)}{m^{4} n^{2}} s t,
$$

$$
\begin{aligned}
V_{26} / g= & \frac{h k\left(h^{2}-k^{2}\right)\left(h^{2}+k^{2}+4 l^{2}\right)}{4 m^{3} n^{3}}\left(c^{2}-t^{2}\right) \\
& +\frac{l\left(-9 h^{4} k^{2}+h^{4} l^{2}-9 h^{2} k^{4}+k^{4} l^{2}-6 h^{2} k^{2} l^{2}-h^{6}-k^{6}\right)}{2 m^{4} n^{3}} c t,
\end{aligned}
$$

$$
\begin{gathered}
V_{27} / g=\frac{l\left(-9 h^{4} k^{2}+h^{4} l^{2}-9 h^{2} k^{4}+k^{4} l^{2}-6 h^{2} k^{2} l^{2}-h^{6}-k^{6}\right)}{4 m^{4} n^{3}}\left(c^{2}\right. \\
\left.-t^{2}\right)-\frac{h k\left(h^{2}-k^{2}\right)\left(h^{2}+k^{2}+4 l^{2}\right)}{2 m^{3} n^{3}} c t, \quad \text { (A120) } \\
V_{31} / g=\frac{2\left(h^{2} k^{2}+h^{2} l^{2}+k^{2} l^{2}\right)}{m^{4}} s t, \quad \text { (A121) } \\
V_{32} / g=\frac{l\left(h^{2} l^{2}+k^{2} l^{2}-h^{4}-k^{4}\right)}{4 m^{4} n}\left(4 s^{2}-c^{2}-3 t^{2}\right) \\
+\frac{h k\left(h^{2}-k^{2}\right)}{2 m^{3} n} c t, \\
V_{33} / g=\frac{h k\left(h^{2}-k^{2}\right)}{4 m^{3} n}\left(4 s^{2}-c^{2}-3 t^{2}\right) \\
-\frac{l\left(h^{2} l^{2}+k^{2} l^{2}-h^{4}-k^{4}\right)}{2 m^{4} n} c t,
\end{gathered}
$$

$$
V_{34} / g=\frac{2 h k l\left(h^{2}-k^{2}\right)}{m^{3} n^{2}} s c-\frac{2\left(h^{4} k^{2}+h^{2} k^{4}-h^{4} l^{2}-k^{4} l^{2}\right)}{m^{4} n^{2}} s t,
$$

$$
V_{35} / g=-\frac{2\left(h^{4} k^{2}+h^{2} k^{4}-h^{4} l^{2}-k^{4} l^{2}\right)}{m^{4} n^{2}} s c-\frac{2 h k l\left(h^{2}-k^{2}\right)}{m^{3} n^{2}} s t,
$$

$$
\begin{aligned}
V_{36} / g= & \frac{l\left(-9 h^{4} k^{2}+h^{4} l^{2}-9 h^{2} k^{4}+k^{4} l^{2}-6 h^{2} k^{2} l^{2}-h^{6}-k^{6}\right)}{4 m^{4} n^{3}}\left(c^{2}\right. \\
& \left.-t^{2}\right)-\frac{h k\left(h^{2}-k^{2}\right)\left(h^{2}+k^{2}+4 l^{2}\right)}{2 m^{3} n^{3}} c t, \\
V_{37} / g= & -\frac{h k\left(h^{2}-k^{2}\right)\left(h^{2}+k^{2}+4 l^{2}\right)}{4 m^{3} n^{3}}\left(c^{2}-t^{2}\right) \\
& -\frac{l\left(-9 h^{4} k^{2}+h^{4} l^{2}-9 h^{2} k^{4}+k^{4} l^{2}-6 h^{2} k^{2} l^{2}-h^{6}-k^{6}\right)}{2 m^{4} n^{3}} c t .
\end{aligned}
$$

\section{APPENDIX B: SPECIFIC CASE OF TRANSMISSION GEOMETRY AND $(11 N)$ crystal face}

The equations in Appendix A are now given for the specific case of normal incidence for a $(11 N)$ crystal face: $h$ $=k=1, l=N$, and $\phi_{\mathrm{NIR}}=\Phi=0$ : 


$$
\begin{aligned}
& m=\sqrt{2+N^{2}} \\
& n=\sqrt{2} \text {, } \\
& s=0, \\
& c=1 \text {, } \\
& t=0, \\
& d=d_{14} E_{0}^{2}, \\
& g=\gamma^{\prime} F_{0} E_{0}^{2}, \\
& A_{11}=\frac{1}{\sqrt{2+N^{2}}}, \\
& A_{12}=-\frac{1}{\sqrt{2}} \text {, } \\
& A_{13}=-\frac{N}{\sqrt{2+N^{2}} \sqrt{2}}, \\
& A_{21}=\frac{1}{\sqrt{2+N^{2}}}, \\
& A_{22}=\frac{1}{\sqrt{2}} \text {, } \\
& A_{23}=-\frac{N}{\sqrt{2+N^{2}} \sqrt{2}}, \\
& A_{31}=\frac{N}{\sqrt{2+N^{2}}}, \\
& A_{32}=0 \text {, } \\
& A_{33}=\frac{\sqrt{2}}{\sqrt{2+N^{2}}}, \\
& B_{11}=0 \text {, } \\
& B_{12}=-\frac{1}{\sqrt{2}}, \\
& B_{13}=-\frac{N}{\sqrt{2+N^{2}} \sqrt{2}}, \\
& B_{21}=0 \text {, } \\
& B_{22}=\frac{1}{\sqrt{2}} \text {, }
\end{aligned}
$$$$
B_{23}=-\frac{N}{\sqrt{2+N^{2}} \sqrt{2}},
$$

$$
B_{31}=0
$$$$
B_{32}=0
$$

$$
B_{33}=\frac{\sqrt{2}}{\sqrt{2+N^{2}}},
$$

$$
C_{11} / d=-\frac{N}{2+N^{2}},
$$$$
C_{12} / d=0
$$$$
C_{13} / d=0
$$

$$
C_{14} / d=\frac{N}{2+N^{2}},
$$$$
C_{15} / d=\frac{1}{\sqrt{2+N^{2}}},
$$$$
C_{21} / d=-\frac{N}{2+N^{2}},
$$$$
C_{22} / d=0
$$$$
C_{23} / d=0
$$

$$
C_{24} / d=\frac{N}{2+N^{2}},
$$

$$
C_{25} / d=-\frac{1}{\sqrt{2+N^{2}}},
$$

$$
C_{31} / d=-\frac{1}{2+N^{2}},
$$

$$
C_{32} / d=0
$$$$
C_{33} / d=0,
$$

$$
C_{34} / d=-\frac{1+N^{2}}{2+N^{2}},
$$

$$
C_{35} / d=0,
$$

$$
D_{11} / d=-\frac{3 N}{\left(2+N^{2}\right)^{3 / 2}},
$$

$$
D_{12} / d=0 \text {, }
$$




$$
\begin{aligned}
& D_{13} / d=0, \\
& D_{14} / d=\frac{N\left(1-N^{2}\right)}{\left(2+N^{2}\right)^{3 / 2}}, \\
& D_{15} / d=0, \\
& D_{21} / d=0 \text {, } \\
& D_{22} / d=0, \\
& D_{23} / d=0 \text {, } \\
& D_{24} / d=0 \text {, } \\
& D_{25} / d=-\frac{2}{\sqrt{2+N^{2}} \sqrt{2}}, \\
& D_{31} / d=-\frac{\left(2-2 N^{2}\right)}{\left(2+N^{2}\right)^{3 / 2} \sqrt{2}}, \\
& D_{32} / d=0 \text {, } \\
& D_{33} / d=0 \text {, } \\
& D_{34} / d=-\frac{\left(2+4 N^{2}\right)}{\left(2+N^{2}\right)^{3 / 2} \sqrt{2}}, \\
& D_{35} / d=0 \text {, } \\
& G_{11} / d=-\frac{3 N}{\left(2+N^{2}\right)^{3 / 2}}, \\
& G_{12} / d=0, \\
& G_{13} / d=0, \\
& G_{14} / d=\frac{N\left(1-N^{2}\right)}{\left(2+N^{2}\right)^{3 / 2}}, \\
& G_{15} / d=0 \\
& G_{16} / d=0 \\
& G_{17} / d=0, \\
& G_{21} / d=0, \\
& G_{22} / d=0, \\
& G_{23} / d=-\frac{3\left(1-N^{2}\right)}{\left(2+N^{2}\right)^{3 / 2} \sqrt{2}}, \\
& G_{24} / d=0,
\end{aligned}
$$

$$
\begin{gathered}
G_{25} / d=0, \\
G_{26} / d=0, \\
G_{27} / d=-\frac{3\left(1+N^{2}\right)}{\left(2+N^{2}\right)^{3 / 2} \sqrt{2}}, \\
G_{31} / d=0, \\
G_{32} / d=-\frac{\left(1-N^{2}\right)}{\left(2+N^{2}\right)^{3 / 2} \sqrt{2}},
\end{gathered}
$$$$
G_{33} / d=0,
$$$$
G_{34} / d=0,
$$$$
G_{35} / d=0 \text {, }
$$$$
G_{36} / d=-\frac{3\left(1+N^{2}\right)}{\left(2+N^{2}\right)^{3 / 2} \sqrt{2}},
$$$$
G_{37} / d=0,
$$$$
T_{11} / g=\frac{1+N^{2}}{2\left(2+N^{2}\right)^{3 / 2}},
$$$$
T_{12} / g=0
$$$$
T_{13} / g=0
$$$$
T_{14} / g=\frac{1}{2\left(2+N^{2}\right)^{3 / 2}},
$$$$
T_{15} / g=\frac{N}{2\left(2+N^{2}\right)},
$$$$
T_{21} / g=\frac{1+N^{2}}{2\left(2+N^{2}\right)^{3 / 2}},
$$$$
T_{22} / g=0
$$$$
T_{23} / g=0,
$$$$
T_{24} / g=\frac{1}{2\left(2+N^{2}\right)^{3 / 2}},
$$$$
T_{25} / g=-\frac{N}{2\left(2+N^{2}\right)},
$$$$
T_{31} / g=\frac{N}{\left(2+N^{2}\right)^{3 / 2}},
$$$$
T_{32} / g=0
$$ 


$$
\begin{aligned}
& T_{33} / g=0, \\
& T_{34} / g=-\frac{N}{\left(2+N^{2}\right)^{3 / 2}}, \\
& T_{35} / g=0, \\
& U_{11} / g=\frac{1+2 N^{2}}{\left(2+N^{2}\right)^{2}}, \\
& U_{12} / g=0, \\
& U_{13} / g=0, \\
& U_{14} / g=\frac{1-N^{2}}{\left(2+N^{2}\right)^{2}}, \\
& U_{15} / g=0, \\
& U_{21} / g=0, \\
& U_{22} / g=0, \\
& U_{23} / g=0, \\
& U_{24} / g=0, \\
& U_{25} / g=-\frac{N}{\left(2+N^{2}\right) \sqrt{2}}, \\
& U_{31} / g=\frac{N\left(1-N^{2}\right)}{\left(2+N^{2}\right)^{2} \sqrt{2}}, \\
& U_{32} / g=0, \\
& U_{33} / g=0, \\
& U_{34} / g=\frac{-3 N}{\left(2+N^{2}\right)^{2} \sqrt{2}}, \\
& U_{35} / g=0, \\
& V_{11} / g=\frac{1+2 N^{2}}{\left(2+N^{2}\right)^{2}},
\end{aligned}
$$

$$
\begin{aligned}
& V_{12} / g=0, \\
& V_{13} / g=0, \\
& V_{14} / g=\frac{1-N^{2}}{\left(2+N^{2}\right)^{2}}, \\
& V_{15} / g=0, \\
& V_{16} / g=0, \\
& V_{17} / g=0, \\
& V_{21} / g=0, \\
& V_{22} / g=0, \\
& V_{23} / g=\frac{3 N\left(1-N^{2}\right)}{2 \sqrt{2}\left(2+N^{2}\right)^{2}}, \\
& V_{24} / g=0, \\
& V_{25} / g=0 \text {, } \\
& V_{26} / g=0 \text {, } \\
& V_{27} / g=\frac{-N\left(5+N^{2}\right)}{2 \sqrt{2}\left(2+N^{2}\right)^{2}}, \\
& V_{31} / g=0, \\
& V_{32} / g=\frac{N\left(1-N^{2}\right)}{2 \sqrt{2}\left(2+N^{2}\right)^{2}}, \\
& V_{33} / g=0, \\
& V_{34} / g=0, \\
& V_{35} / g=0, \\
& V_{36} / g=\frac{-N\left(5+N^{2}\right)}{2 \sqrt{2}\left(2+N^{2}\right)^{2}}, \\
& V_{37} / g=0 .
\end{aligned}
$$

${ }^{1}$ B. Ferguson and X.-C. Zhang, Nature Mater. 1, 26 (2002).

${ }^{2}$ P. H. Siegel, IEEE Trans. Microwave Theory Tech. 50, 910 (2002).

${ }^{3}$ P. LeFur and D. H. Auston, Appl. Phys. Lett. 28, 21 (1976).

${ }^{4}$ Y. Cai, I. Brener, J. Lopata, J. Wynn, L. Pfeiffer, and J. Federici,
Appl. Phys. Lett. 71, 2076 (1997).

${ }^{5}$ C. Sydlo, R. Mendis, J. Sigmund, M. Feiginov, H. L. Hartnagel, and P. Meissner, IEEE/ACES International Conference on Wireless Communications and Applied Computational Electromagnetics, 2005, pp. 873-877. 
${ }^{6}$ G. Zhao, R. N. Schouten, N. van der Valk, W. T. Wenckebach, and P. C. M. Planken, Phys. Med. Biol. 47, 3699 (2002).

${ }^{7}$ D. Schoenherr, H. L. Hartnagel, S. Hargreaves, R. A. Lewis, and M. Henini, Semicond. Sci. Technol. 23, 105012 (2008).

${ }^{8}$ S. L. Chuang, S. Schmitt-Rink, B. I. Greene, P. N. Saeta, and A. F. J. Levi, Phys. Rev. Lett. 68, 102 (1992).

${ }^{9}$ B. I. Greene, P. N. Saeta, D. R. Dykaar, S. Schmitt-Rink, and S. L. Chuang, IEEE J. Quantum Electron. 28, 2302 (1992).

${ }^{10}$ M. Nakajima, Y. Oda, and T. Suemoto, Quantum Electronics Conference, 2005 International, 2005, pp. 1340-1341.

${ }^{11}$ P. Gu, M. Tani, S. Kono, K. Sakai, and X.-C. Zhang, J. Appl. Phys. 91, 5533 (2002).

${ }^{12} \mathrm{X}$. Mu, I. B. Zotova, and Y. J. Ding, IEEE J. Sel. Top. Quantum Electron. 14, 315 (2008).

${ }^{13}$ D. You, R. R. Jones, P. H. Bucksbaum, and D. R. Dykaar, J. Opt. Soc. Am. B 11, 486 (1994).

${ }^{14}$ A. Rice, Y. Jin, X. F. Ma, X.-C. Zhang, D. Bliss, J. Larkin, and M. Alexander, Appl. Phys. Lett. 64, 1324 (1994).

${ }^{15}$ Y. H. Jin and X.-C. Zhang, J. Nonlinear Opt. Phys. Mater. 4, 459 (1995).

${ }^{16}$ Q. Chen, M. Tani, Z. Jiang, and X.-C. Zhang, J. Opt. Soc. Am. B 18, 823 (2001).

${ }^{17}$ S. Kono, P. Gu, M. Tani, and K. Sakai, Appl. Phys. B: Lasers
Opt. 71, 901 (2000).

${ }^{18}$ M. Reid, I. V. Cravetchi, and R. Fedosejevs, Phys. Rev. B 72, 035201 (2005).

${ }^{19}$ V. L. Malevich, A. Krotkus, A. Bičiūnas, and V. Pačebutas, J. Appl. Phys. 104, 113117 (2008).

${ }^{20}$ J. B. Khurgin, J. Opt. Soc. Am. B 11, 2492 (1994).

${ }^{21}$ F. Nastos and J. E. Sipe, Phys. Rev. B 74, 035201 (2006).

${ }^{22}$ M. Bieler, K. Pierz, U. Siegner, and P. Dawson, Phys. Rev. B 76, 161304(R) (2007).

${ }^{23}$ W. I. Wang, E. E. Mendez, T. S. Kuan, and L. Esaki, Appl. Phys. Lett. 47, 826 (1985).

${ }^{24}$ S. S. Bose, B. Lee, M. H. Kim, G. E. Stillman, and W. I. Wang, J. Appl. Phys. 63, 743 (1988).

${ }^{25}$ D. K. Biegelsen, R. D. Bringans, J. E. Northrup, and L.-E. Swartz, Phys. Rev. Lett. 65, 452 (1990).

${ }^{26}$ M. Wassermeier, J. Sudijono, M. D. Johnson, K. T. Leung, B. G. Orr, L. Däweritz, and K. Ploog, Phys. Rev. B 51, 14721 (1995).

${ }^{27}$ P. Moriarty et al., Phys. Rev. B 55, 15397 (1997).

${ }^{28}$ P. Moriarty, P. H. Beton, and D. A. Woolf, Phys. Rev. B 51, 7950 (1995).

${ }^{29}$ T. A. Germer, K. W. Kołasiński, J. C. Stephenson, and L. J. Richter, Phys. Rev. B 55, 10694 (1997). 